\title{
A Novel Agent-Based Framework in Bridge-Mode Hypervisors of Cloud Security
}

M. Janbeglou and W. Yan

AUT University, NZ 


\section{Content}

- Cloud computing

- Virtualization and its risks

- Proposed Virtual Network Model

- Evaluations

- Conclusion 


\section{Cloud Computing}

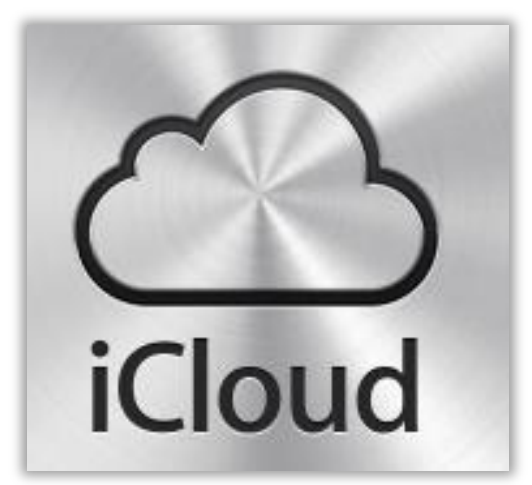

KMO 2012, Spain 

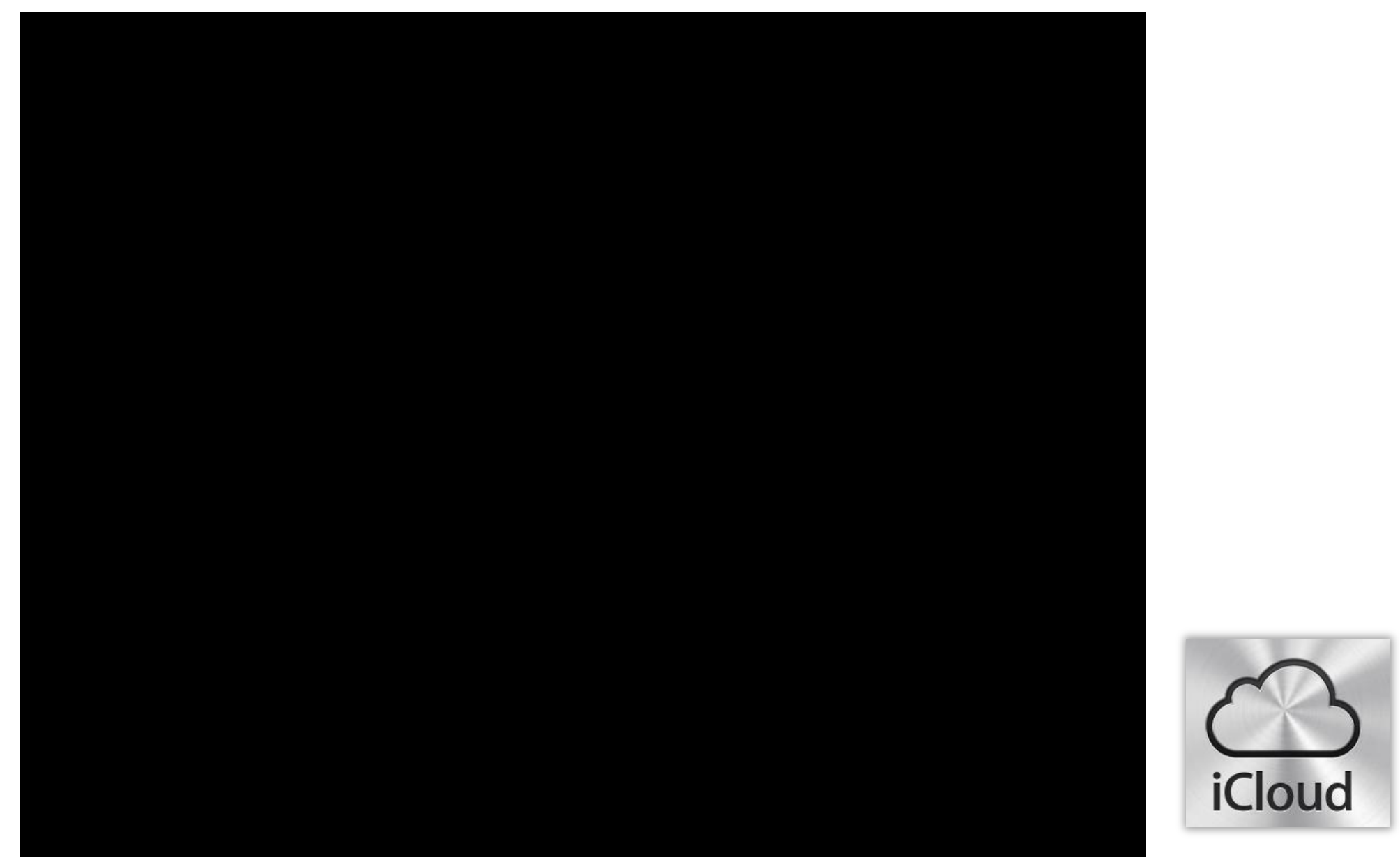

KMO 2012, Spain 


\section{Cloud Computing (NIST,USA)}

Cloud computing is a model for enabling convenient, on-demand network access to a shared pool of configurable computing resources (i.e. networks, servers, storage, applications, and services) that can be rapidly provisioned and released with minimal management effort or service provider interaction. 


\section{Cloud Advantages}

- On-demand

- Self-service

- Location independent

- Elastic

- Accessible 


\section{Clouds}

- Private Cloud: This model is for usage of individual organization and not shared among other organizations.

- Community Cloud: This model is shared with several set of organizations.

- Public Cloud: The most common type of cloud infrastructure that is made to be available for public.

- Hybrid Cloud: This model is made by combining two or more deployment models (private, community, or public). 


\section{Cloud Components}

- SaaS: Software as a Service

- PaaS: Platform as a Service

- laaS: Infrastructure as a Service 


\section{Virtualization and Its Risks}

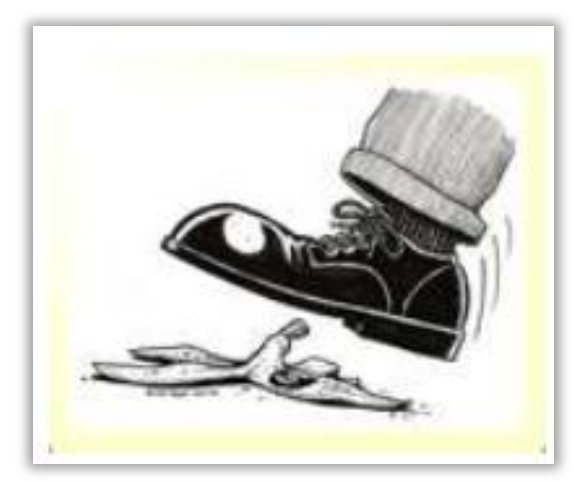

KMO 2012, Spain 


\section{Virtualization}

- Visualization provides the ability of installing multiple OSs on different VMs on a same physical machine and as a result it increases the machine utilization.

- Virtualization is responsible for splitting resources on a single physical machine into multiple VMs.

- Virtualization helps Cloud Service Providers (CSP) to solve the complexity issues in delivering services, managing shared resources and utilizations, isolating VMs, and providing security. 


\section{Risks Towards Virtualization}

- Virtualized systems risks

- Hypervisor risks

- Virtual machine risks

- Virtual network risks 


\section{Virtualized System Risks}

- Visualization makes the security management more complex.

- Visualization needs more controlling and monitoring of the shared resources.

- Larger security threats arise when many VMs are combined into a physical machine.

- Systems are dynamic and flexible to changes, defining security boundaries will be complicated. 


\section{Hypervisor Risks}

- Hypervisor is a software program, it is inherently vulnerable to the growth of volume and complexity of application codes.

- Hypervisor provides physical server resources sharing and $\mathrm{VM} /$ host isolation.

- Vulnerabilities in current hypervisors are Rogue Hypervisors, External Modification of the Hypervisor, VM Escape, and Denial-of-Service. 


\section{Virtual Machine Risks}

- Use the shared resources on a physical server to deliver business needs.

$>$ Working on a same physical machine

$>$ Using the shared resources 


\section{Virtual Machine Risks}

- Shared clipboard attack

- Keystroke logging attack

- Monitoring VMs from an infected host 


\section{Virtual Network Risks}

- In physical networks, firewall and encryptions mechanisms are the main tools for applying security.

- In virtual networks, almost all the physical network threats are likely to happen.

- Isolation does a similar function in virtual networks. 


\section{Virtual Network Model}

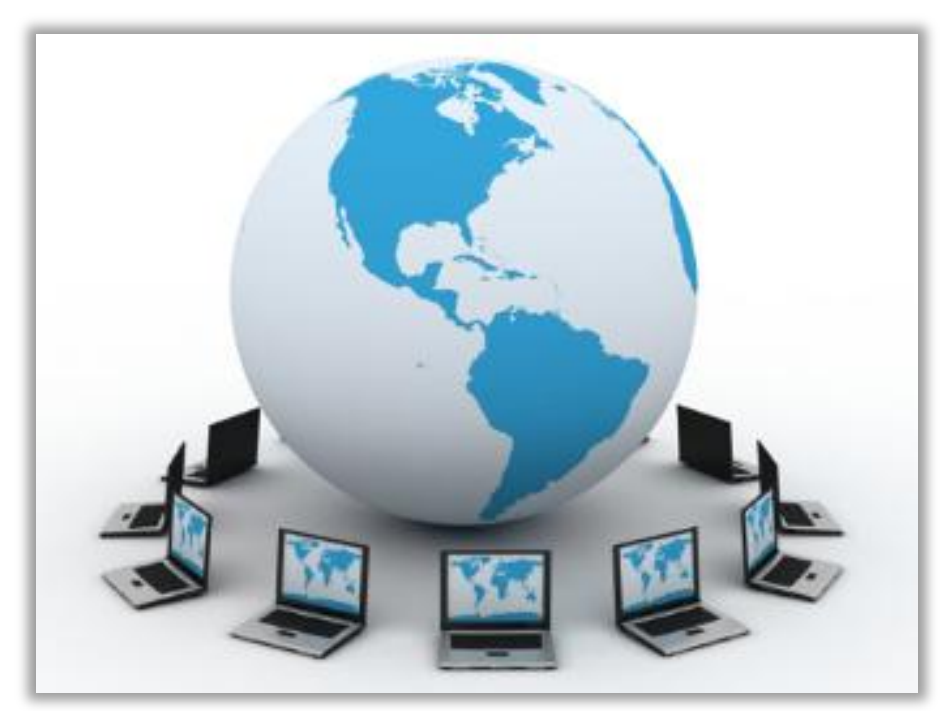

KMO 2012, Spain 


\section{Our Contributions}

- This paper proposes a model to improve the laaS security on the shared network resources by making VMs invisible from attackers, and as a result, preventing them from performing the key step of network-related attacks. 


\section{Proposed Agent}

- The proposed model introduce an agent to provide a centralized virtual network management for all of the VMs residing in a physical server.

- The agent is to help the hypervisor to provide security by confining the visibility and accessibility of the VMs network resources. 


\section{Proposed Model Steps}

1) Generating network-sub-interfaces

and Random IP address configurations

2) Generating PPTP (Point2Point Tunneling Protocol)

configurations

3) Customizing the packet-filtering 


\section{Network Sub-interfaces}

- Network sub-interfaces refers to sub-interfaces that are created from VM Interface.

- Each of the sub-interfaces will be assigned to corresponding VMs.

- Randomly generated IP address configurations are assigned to the VMs by a DHCP (Dynamic Host Configuration Protocol) service running on the agent. 


\section{Generating IP addresses in PPTP service}

- Counting the number of domains available.

- Counting the maximum number of VMs in each domain.

- Calculating appropriate subnet for each domain. The appropriate subnet is the smallest possible subnet to cover a group of VMs working in a domain.

- Generating and assigning IPs to each VM and network-sub-interface. 


\section{Customizing the Packet-Filtering}

- Packet-filtering improves the security of the whole system by confining any internal-communications via dropping packets originating from internal VMs residing in different domains.

- Because the packet-filtering bans any VMs intercommunications within different domains, the VMs' IP addresses remain invisible for attackers. 


\section{Evaluations}

- CIA: Confidentiality, Integrity, and Availability.

- AAA: Authentication, Authorization, and Accountability.

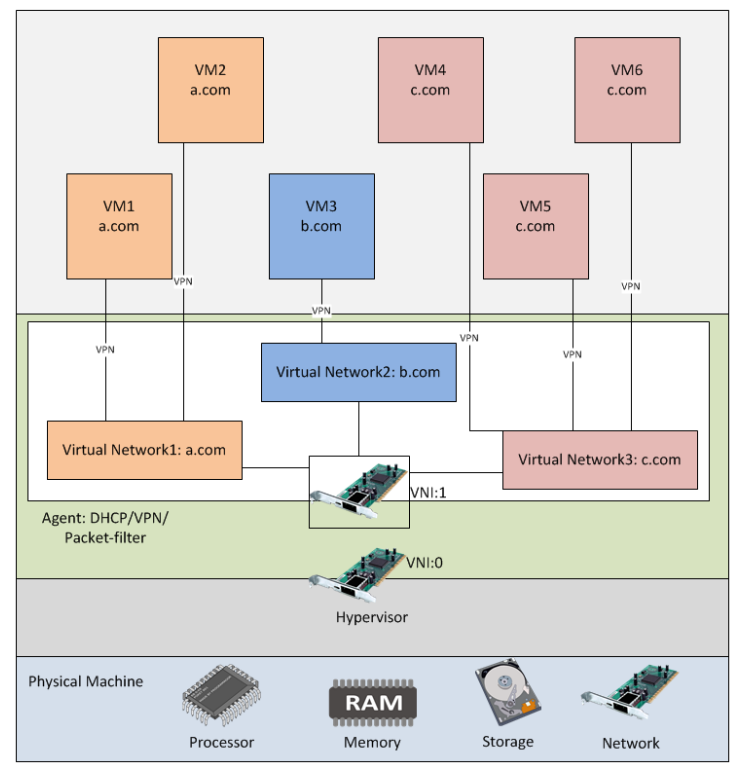

KMO 2012, Spain 


\section{Conclusion}

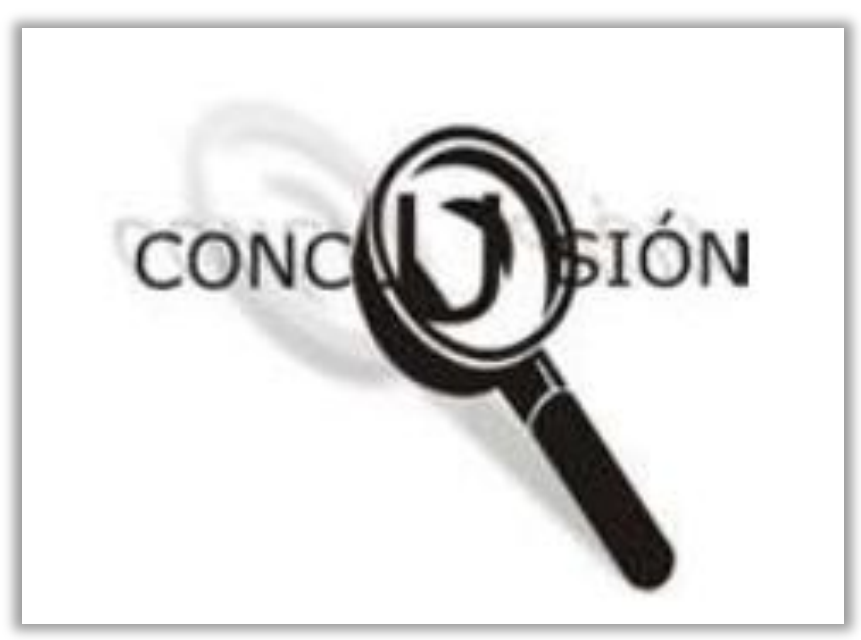

KMO 2012, Spain 


\section{Conclusion}

1) Security risks towards each virtualized system component have been examined.

2) A proposed model was introduced.

3) The evaluation of proposed model was addressed. 


\section{A Novel Agent-Based Framework in Bridge-Mode Hypervisors of Cloud Security}

M. Janbeglou and W. Yan

AUT University, NZ 